\title{
Defacing biases manual and automated quality assessments of structural MRI with MRIQC \\ Céline Provins ${ }^{1}$, Yasser Alemán-Gómez ${ }^{1,2}$, Martine Cleusix², Raoul Jenni², Jonas Richiardi', Patric Hagmann ${ }^{1}$, Oscar Esteban ${ }^{1}$ \\ ${ }^{1}$ Department of Radiology, Lausanne University Hospital and University of Lausanne, Lausanne, Switzerland
}

${ }^{2}$ Center for Psychiatric Neuroscience, Department of Psychiatry, Lausanne University Hospital and University of Lausanne. Prilly, Switzerland.

\section{Introduction}

Defacing (i.e. removing facial features) from structural imaging has become a necessary step before data sharing to ensure participants' anonymity (Schwarz et al. 2021; Fig 1A). This process has proven to have some deleterious effects on the downstream research workflow (de Sitter et al. 2020). Here, we present an exploratory analysis prior to testing the hypothesis that both quality ratings by human experts and the image quality metrics (IQMs) that MRIQC (Esteban et al. 2017) extracts are affected by defacing. We found sufficient evidence on a small sample that there might be an effect. Therefore, we will pre-register and carry out a confirmatory analysis on a larger, unseen, sample.

\section{Methods}

We use the T1-weighted ( $\mathrm{T} 1 \mathrm{w}$ ) images corresponding to 10 healthy subjects (5 Males/5 Females) randomly drawn from the Treatment and Early Intervention in Psychosis Program (TIPP, Lausanne University Hospital), a dataset where facial features had not been removed ("non-defaced" data). MRI sessions were performed on a 3T Magnetom PRISMA, Siemens Medical Solutions, equipped with a 32-channel head coil each. Each scanning session included a magnetization prepared rapid acquisition gradient echo (MPRAGE) with echo time $=2.98 \mathrm{~ms}$, repetition time $=2300 \mathrm{~ms}$, inversion time $=900 \mathrm{~ms}$, flip angle $=8^{\circ}$, field of view $=160 \times 240 \times 256 \mathrm{~mm}^{3}$ and voxel size $=1 \times 1 \times 1.2 \mathrm{~mm}^{3}$.

First, a "defaced" dataset was generated using PyDeface (Gulban et al. 2019). The dataset was then blinded by reassigning 20 randomly drawn subject identifiers, and MRIQC was executed on all the non-defaced/defaced T1w images. To investigate the impact of defacing on manual assessments, two trained raters (authors $\mathrm{CP}$ and $\mathrm{OE}$ ) assigned each image a quality grade (1-exclude, 2-poor, 3-good, 4-excellent) using the visual reports generated by MRIQC. We report Bland-Altman (BA) plots showing the reliability of the human raters (Fig.1C) and the IQMs automatically generated by MRIQC (Fig.2). For readability of the figure, only 5 IQMs are reported here. Those were manually selected as IQMs, whose definition is susceptible to defacing influence, and that present large differences. The figure with all IQMs can be found online (Provins 2021). Finally, we determined whether there are group differences on the selected five IQMs between the two conditions (non-defaced/defaced) with a MANOVA (Multivariate analysis of variance).

\section{Results}

We found differences between the manual quality assessments of non-defaced and defaced images. Overall, the defaced image is often being judged as having better quality. Fig 1B illustrates a 
leading reason why the junior rater's consistency was low : one of the main information used for image quality judgment, that is eye spillover, is removed by defacing. The expert rater seems more reliable in grading the same image with and without face, as a higher number of subjects lie on the zero-difference reference line on the BA plot (Fig. 1C), but not completely reliable as $40 \%$ of the subjects were still judged of higher quality when defaced. In conclusion, these observations support the fact that defacing altered the raters' assessments.

IQMs also varied after defacing. Consistently with the BA plots presented in Fig. 2, MANOVA reveals a significant difference between the datasets with and without face ( $\left.F_{(5,14)}=8.269, p<.001\right)$ meaning that defacing has an effect on at least one of the 5 selected IQMs. To note, this analysis does not inform about the direction of change or the importance of each of the IQMs. Those questions will be addressed in our upcoming pre-registered study.

\section{Conclusions}

Both manual quality assessments and IQMs showed disagreement between the dataset non-defaced and defaced, as investigated with BA plots. Results suggest that raters perceive defaced images as having better quality overall. Moreover, a MANOVA revealed that one or more IQMs are significantly influenced by defacing. We conclude that a pre-registered, confirmatory study on a larger database is justified.
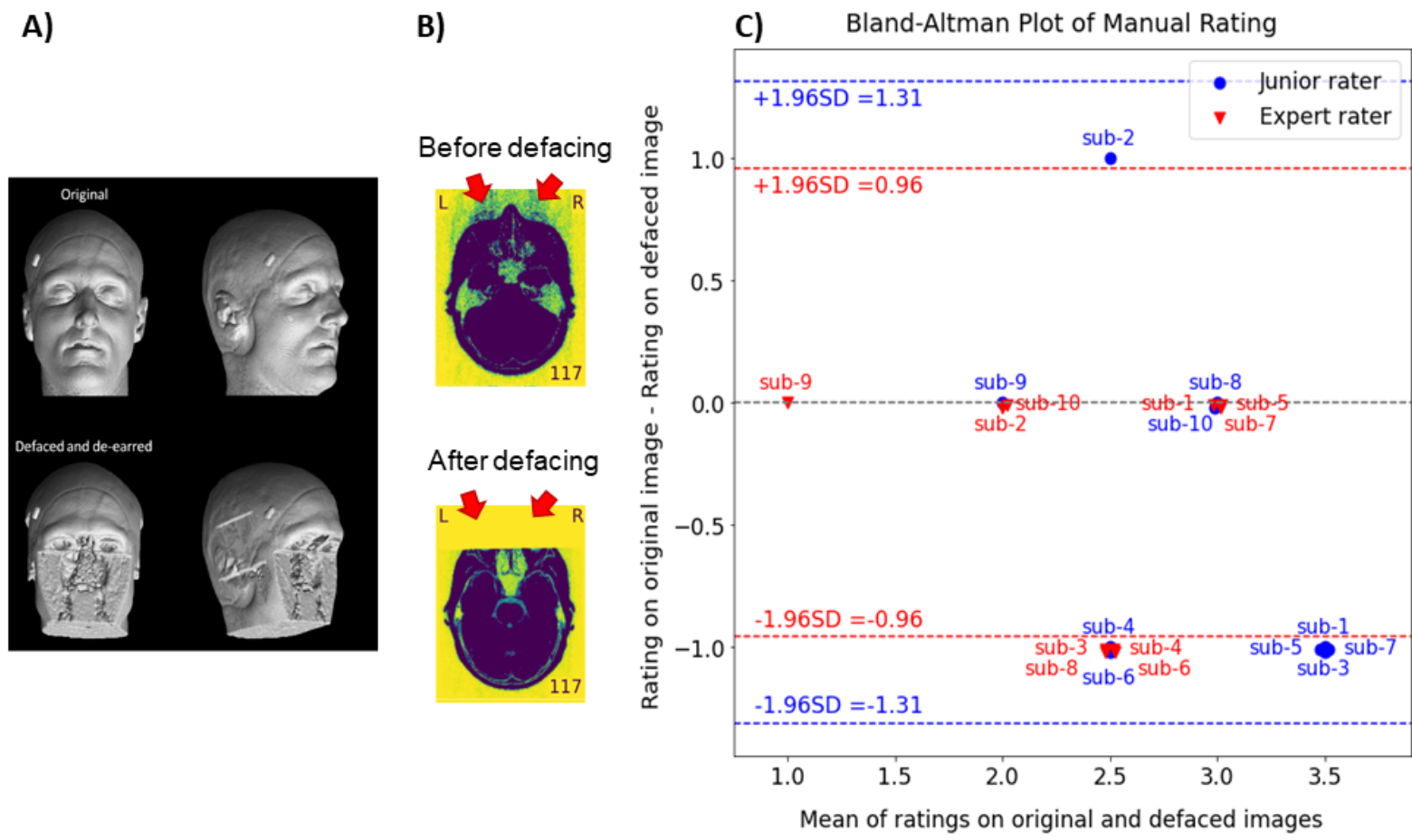

Fig. 1. Manual quality assessment is influenced by defacing. A) An example of volume-rendered T1w image before and after defacing (adapted from (Theyers et al. 2021)) B) Eye spillover is an example of information used for rating image quality that is removed by defacing. C) The Bland-Altman plot shows the evolution of the rating before vs after defacing. The gray dashed line corresponds to the rater attributing the same quality score between the image with and without face. The plot suggests that a less experienced rater may be more influenced by the defacing. 
Bland-Altman Plot

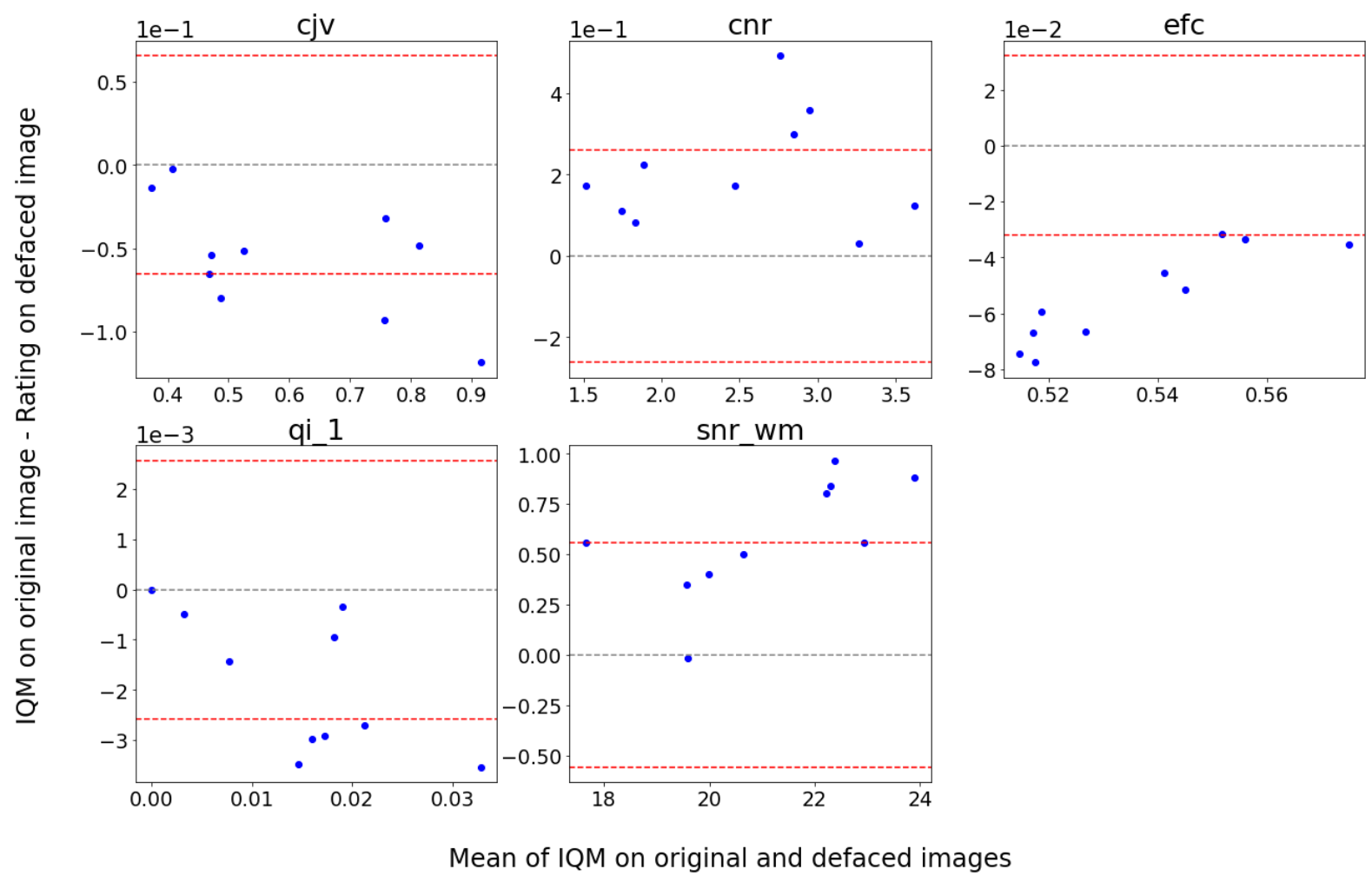

Fig.2 IQMs vary after defacing. Bland-Altman plots for a selection of IQMs. The grey line lies at 0 and represents the ideal condition where the IQM value would be identical between the image with and without face. This means that if the points are concentrated above or below the grey line, the IQM is biased by defacing. The red lines correspond to the $95 \%$ confidence interval centered around the zero-difference reference line, specifically $0 \pm 1.96 * S D$.

\section{Acknowledgments}

This work has been supported by the NIMH (RF1MH121867; OE). CP, and OE receive support from the SNSF Ambizione Project "Uncovering The Interplay Of Structure, Function, and Dynamics of Brain Connectivity using MRI” (grant number 185872).

\section{References}

Esteban, Oscar, Daniel Birman, Marie Schaer, Oluwasanmi O. Koyejo, Russell A. Poldrack, and Krzysztof J. Gorgolewski. 2017. "MRIQC: Advancing the Automatic Prediction of Image Quality in MRI from Unseen Sites." Edited by Boris C Bernhardt. PLOS ONE 12 (9): e0184661-e0184661. https://doi.org/10.1371/journal.pone.0184661.

Gulban, Omer Faruk, Dylan Nielson, Russ Poldrack, John Lee, Chris Gorgolewski, Vanessasaurus, and Satrajit Ghosh. 2019. Poldracklab/Pydeface: V2.0.0. Zenodo. https://doi.org/10.5281/zenodo.3524401.

Provins, Céline. 2021. "Defacing Biases Manual and Automated Quality Assessments of Structural MRI with MRIQC," December.

https://mfr.osf.io/render?url=https://osf.io/jfsut/?direct\%26mode=render\%26action=downlo ad\%26mode=render.

Schwarz, Christopher G., Walter K. Kremers, Heather J. Wiste, Jeffrey L. Gunter, Prashanthi Vemuri, Anthony J. Spychalla, Kejal Kantarci, et al. 2021. "Changing the Face of Neuroimaging Research: Comparing a New MRI de-Facing Technique with Popular Alternatives." 
Neurolmage 231 (May): 117845. https://doi.org/10.1016/j.neuroimage.2021.117845.

Sitter, A. de, M. Visser, I. Brouwer, K. S. Cover, R. A. van Schijndel, R. S. Eijgelaar, D. M. J. Müller, et al. 2020. "Facing Privacy in Neuroimaging: Removing Facial Features Degrades Performance of Image Analysis Methods." European Radiology 30 (2): 1062-74. https://doi.org/10.1007/s00330-019-06459-3.

Theyers, Athena E., Mojdeh Zamyadi, Mark O'Reilly, Robert Bartha, Sean Symons, Glenda M. MacQueen, Stefanie Hassel, et al. 2021. "Multisite Comparison of MRI Defacing Software Across Multiple Cohorts." Frontiers in Psychiatry 12: 189. https://doi.org/10.3389/fpsyt.2021.617997. 